\title{
Differentiated Tasks System in Math as a Tool to Develop University Students' Learning Motivation
}

\author{
Ramis R. Nasibullov \\ Kazan (Volga region) Federal University, RUSSIA \\ Aliya V. Konysheva \\ Vyatka State University of Humanities, RUSSIA \\ Viya G. Ignatovich \\ Belorussian State Pedagogical University, BELORUSSIA
}

•Received 22 June $2015 \bullet$ Revised 22 August $2015 \bullet$ Accepted 23 September 2015

The relevance of the study is due to the direction of the modern education model on the formation of a competitive creative personality, with the need for continuous selfimprovement and self-development. It should be emphasized that the effectiveness of training students is largely determined by the level of motivation, interest, and the subjects' personal involvement. Therefore, the article is aimed at the disclosure of the mechanisms of development of learning motivation of students through the system of differentiated tasks. The leading approach to the study of the problem was the personalactive approach, which aims to reveal the discussed question from the perspective of dialogism, subjectivity and individuality. The article presents the characteristics of the developed and proven system of differentiated tasks in mathematics, focused on the development of learning motivation of students, as well as providing empirical evidence about the results of implementation. The article describes the principles on which we identified three levels of tasks in mathematics: "algorithms", "problem-search tasks", and "creative tasks". The article may be useful to teachers of higher education.

Keywords: mathematical training, differentiated tasks, learning motivation, system of tasks in mathematics

\section{INTRODUCTION}

\section{Relevance of the problem}

The relevance of the problem of development of learning motivation for students in mathematical training is based on a number of reasons. Firstly, it should be noted that the insufficient level of schooling, and lack of skills of self-organization and self-reflection can cause an adverse psychological situation and, consequently, become a "barrier" mechanism to the study of mathematical disciplines. Secondly, the high degree of abstraction of the studied material, and its poor orientation to the future professional

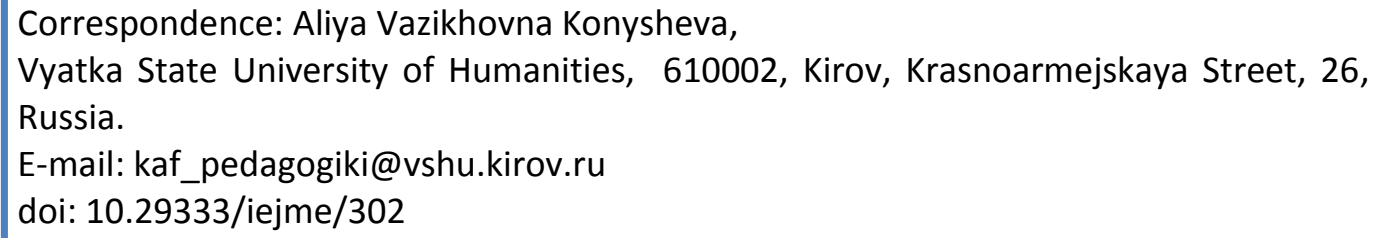


activity leads to a decrease in interest and a corresponding rise in the level of anxiety of students.

Third, the high level of learning motivation is a key factor in improving the mathematical training of students.

\section{Status of the problem}

The current state of the Russian system of higher education leads the researchers' attention to the problem of students' learning motivation development. The Federal Law of the Russian Federation "On Education" emphasized that the presence of positive motivation in students is a prerequisite for effective learning.

Problems of development of learning motivation are reflected in the works of Markova (2010), Serikov (1999), Shchukina (1979) and others. The authors noted a high level of motivation as one of the key conditions to assuring the effectiveness of training.

Learning motivation is characterized by the value orientations, motives and interests, aimed at studying the disciplines reveals the students' willingness to set goals of activities autonomously, to achieve their aspirations, as well as the affirmation to improve their knowledge, skills and abilities.

Considering the development of learning motivation in higher education, we consider it reasonable to characterize students as a special social category. Zimnyaya (1999) notes that the studentship is a period of intense socialization, the development of higher mental functions, formation of the entire intellectual system and the personality as a whole. At the same time, it is student age that has a number of contradictions that are advisable to take into account in the training process between the flowering of intellectual and physical strength, and a limited amount of time, the economic capacity to meet the increased needs; between the desire for independence in the selection of knowledge and techniques and forms of training; between the volume of information and unwillingness to systematize and use it (Overcoming these contradictions becomes feasible, taking into account psycho-physiological and individual personality characteristics. This approach ensures the creation of "the situation of success" for each student, the conditions for realizing the importance of disciplines in the context of future professional activity. It should be noted that "the situation of success" is a purposeful and organized combination of conditions under which the opportunity to achieve significant results in the activity is realized.

Psychological and pedagogical research highlights "the situation of success" contributes to the development of cognitive interest, corrects personality traits (anxiety, insecurity, self-esteem), supports a favorable psychological climate, and significantly increases the learning motivation. We should also point out that motivation is the leading factor that regulates the activities and behavior of the individual. This position is confirmed by the Yerkes-Dodson law, which illustrates the dependence of the result of activities on the degree of motivation.

"No information offered to the student will be assimilated, accepted and perceived, if he/she does not have the appropriate motivation and personal interest in learning and cognitive activity" (Teplov, 1985). It seems appropriate to also pay attention to Teplov's research which indicates that the intellectual abilities of a man are closely linked to the motivational mechanism as they determine motivational sphere and experience its influence. These points bring us to the understanding of the need to ensure a high level of motivation and personal interest of students in the study of mathematical disciplines.

In this context, the view of Serikov (1999) also seems interesting. The authors note that the inclusion of emotional value and personally meaningful elements in the content contributes to the development of inter-subject communication between all participants in the educational process, creates a favorable emotional background and enhances the effectiveness of learning. The material is perceived on a personal level, as if it is experienced as a vital issue. This, in turn, "mobilizes and accordingly develops the 
structure of intelligence". Blonsky (1979) pointed out that "the creation of courses of natural science, mathematics, and physics on the basis of their interests, everyday experience and observations of life ... will contribute to the intellectual development, the formation of abilities to a better understanding of natural phenomena and reality".

\section{Personally-meaningful approach as a methodological basis}

Understanding the above-mentioned findings made it possible to determine the personally-meaningful approach as a methodological basis of development of learning motivation of students in studying mathematics. Such fundamentals are based on the conceptual provisions of person-centered and meaningful approaches. Let's consider the nature of each of these approaches in more details in the context of the problems of the present study.

The recognition of individuality of students, the appeal to their personal experience, their need for self-organization and self-development are the basis of the implementation of a person-centered approach in educational practice. This allowed us to examine the process of development of learning motivation of students from the perspectives of dialogism, subjectivity and individuality. This approach promotes not only the presentation of the content of education in the form of differentiated personcentered tasks, creation of conditions for equal cooperation between teachers and students, but also the development of self-reliance and active learning.

The orientation of teaching on the personality of the student should be considered in close connection with the continuous sequence of formation of methods and techniques for future professional activities. This determines the reasonability of the aspects of the meaningful approach to the problem of development of the educational motivation of students. According to Leontyev (1996), the learning process always implies teaching some activity: object-practical or mental actions. Teaching any kind of activity means making the learning process motivated, teaching students to set certain tasks autonomously, to find ways and means to solve them optimally, organizing their own activities, helping students to form skills of control, and of self-control and selfassessment. This position is not only regarded as the aggregate result of the preparation formed activity-related categories, but also to explore the personality of the teacher and the student as subjects of common activities.

Thus, the meaningful approach allows us to consider the main components of activities performed by the teacher and the student from the common methodological perspective and disclose the nature of their interaction, defines education as a process of continuous change of various activities, makes it possible to characterize the specific features of the activities of all subjects involved in the training process from the perspective of general concepts of the theory of activity in the area of teaching, provides research of pedagogical activity as a category of mutually integrative cooperation of the teacher and the student. This approach aims to regulate the mechanisms of students' learning motivation development.

\section{Functions of mathematical training}

Developing the present idea, we should note that in this study, we consider the process of teaching mathematics to engineering students. Taking into account the specificity of the future work of engineers, we have identified three key approaches to defining the functions of the mathematical training. The first allows us to consider mathematics as an independent science and reveals its methodological potential. In his studies Myshkis (2007) emphasizes that the study of this discipline contributes to the development of "analytical and logical thinking, imagination and spatial representations, algorithmic culture, the formation of the ability to establish causal relationships, to justify claims, to model a situation, encourages creativity and the development of intellectual abilities". From the perspective of this approach the goal of learning 
mathematics is to educate the methodological culture of knowledge of the future professional.

It should be noted that the mathematical discipline cycle is mainly studied in the first and second years of the University. According to Russian scientists, by this time the stage of formation of methodological culture of knowledge of the world is not complete yet. In this context, it seems appropriate to consider the cognitive function of the mathematical training of future engineers.

The use of the second approach, which determines the functionality of mathematics, is caused by its applied orientation and the ability to apply mathematical formalism in solving the problems of the natural sciences. Presented view is the result of understanding and assimilation of various scientific grounds. In particular, its philosophical ideas can be traced in the writings of Hegel (2005), who believed that the "Book of Nature" was written in the mathematical language; the concept of "life-world" of Husserl (1996) highlighting the issue of mathematization of natural sciences and also in modern accounts of Gladun (2004) that reveal the inextricable link between mathematics and the sciences.

The above-mentioned ideas allow considering mathematics and sciences as interlinked and interdependent fields of scientific knowledge. The researchers note that the applicative character of mathematics is realized through the introduction into the system of the preparation of a number of applicative tasks, the content of which reflects the specifics of future professional activity. In particular, Bochkareva (2006) emphasizes that the understanding of the relationship of the mathematical content of professional training is the key to successful learning. This is particularly important since under such an approach, students perceive mathematics not as some kind of "abstract discipline" irrelevant to their future professional activity. From this aspect, the view of Mikhailova (1998) seems to be important. The author systemizes the directions of implementation of the approach discussed: the development and solution of applied problems in accordance with the specifics of their future professional activity, use of mathematical modeling, and the use of technical means of education, among others.

The essence of the third approach is defined as first and second approaches combined. The point of Khinchin (2000) may serve as a common viewpoint on this question. Considering the aims of studying mathematics in higher education, he pointed at their double character expressed in the effort to support the student in mastering the method of higher mathematics as a tool of knowledge and train future specialists to practice mathematical calculations. As a condition to achieve this goal the author identifies the connectivity of each new theory of mind with "adjoining practical calculations".

\section{The system of tasks in mathematical training}

The implementation of the above-mentioned ideas becomes possible through the implementation in the educational practice of the differentiated tasks system, which represents a reasonable set of methodical tasks that differ from each other by levels of difficulty and activities.

Various aspects of this idea have been tested by Russian researchers within different stages of the educational system of the Russian Federation.

Let's define the principles of construction of differentiated tasks defining the development of learning motivation of students. Firstly, to solve problems in mathematics it is advisable to follow the internal logic of the course: learning a new material should comply with the previously acquired knowledge and skills. Secondly, the content should reflect the nature of the tasks of the future professional activity and be personally meaningful for students. Thirdly, the process of solving problems is to meet the principles of accessibility and individuality, and to take into account the personal characteristics of students. Finally, the construction of the system should 
take place taking into account the variability of tasks by type, formulation, methods of solution, increasing complexity and their relationship.

\section{Hypothesis of the study}

Analysis of psycho-pedagogical and methodological literature, as well as studying the experience of other scholars, in developing the discussed problem has shown that the problem of development of learning motivation of students in learning mathematics is to be clarified and further developed. This is primarily due to the change of the educational paradigm, updating methods, and requirements to the level of training of graduates, etc.

The above-mentioned discussion made it possible to formulate the hypothesis of the study: the development of learning motivation of students in teaching mathematics will be more effective if the system of differentiated tasks is developed and implemented.

\section{MATERIALS AND METHODS}

\section{Theoretical and empirical research methods}

We used a range of different methods to carry out the experimental work:

Theoretical methods: analysis, synthesis, classification, comparison, classification, modeling and design;

Empirical methods: study and synthesis of innovative teaching experience of students' mathematical training, questioning, experiment; observation, interview, testing, analysis of students' educational activity products.

\section{Types of differentiated tasks}

The task can be seen as simulated problem situation, involving the person in the cognitive process. It should be emphasized that the tasks in future engineers' training are of key importance. This is due to the fact that, firstly, it ensures the integrity, adaptability and consistency of the educational process. Secondly, in the task solving process students apply the knowledge and expertise, acquire skills of analysis, modeling and forecasting. Applied tasks are of particular importance in the educational practice of engineering students. Tereshin (1990) considers the latter as the objectives set beyond mathematics and solved by mathematical means.

Didactic potential of applied tasks are due to its possibility to emphasize the importance and develop understanding of the value of mathematics and science in the general training of engineers.

Given the above, as the substantive content of the practical component, we considered a complex of differentiated tasks: algorithms, problem-research tasks, and creative tasks.

"Algorithms" are aimed at using rules, formulas and actions undertaken on the model (reproductive level tasks). In addition to formal knowledge of the formulas, definitions and laws, the student should be able to practice the techniques of logical reasoning and evidence. The use of such tasks is very important, because in addition to the formation of certain skills, it helps to create the situation of success and to reduce the level of anxiety of first and second year students.

"Problem-research tasks" aim at developing analytical and research capabilities, skills, process modeling of animate and inanimate nature, interpretation and evaluation of data (reproductive level tasks). Tasks at this level are important because the research process includes a set of three consecutive stages: modeling, direct research and interpretation. These stages correspond with the kinds of future engineer's professional activity. 
Modeling is the construction of a mathematical model of the real situation by transferring specific tasks to the language of mathematics. At this stage, students learn to analyze the situation, highlighting the essential and secondary data relationships, defining their completeness, and describing it using mathematical language.

The second stage includes investigation of the mathematically constructed model and by means of science disciplines. Here, students learn to identify the most appropriate method for the solution of the task, to choose methods and succession of actions, to use mathematical tools, differentiating complicated tasks and simpler subtasks.

Interpretation involves correlation of the result with the original data, in other words; transferring the response and its evaluation in accordance with the specifics of their future professional activity. At this stage, students learn to draw conclusions by solving the task, to conduct a qualitative analysis of the data, and to establish causeand-effect relationships of the results. As a rule, tasks of this type are solved by students with more stable motivation and with rather high level of awareness of mathematical disciplines' importance in their future careers.

Creative tasks include 'open type' tasks or 'partially open type' tasks. Open type tasks have a "blurred" condition from which it is not sufficiently clear how to act, what to use as the solution, but the desired result is clear (Utemov, 2012). These tasks require a variety of solutions, promote the formation of research and reflexive skills, ability to predict. These types of tasks are valuable in the future engineers' training for the reason that allows to realize the idea of heuristic approach to solving the problem, to use a non-standard way of thinking, etc.

As a rule, creative tasks are chosen by students with a high level of motivation, selfcontrol and self-esteem. They demonstrate fluency in mathematical tools and knowledge of sciences, hypothesize in non-standard situation solving, and they are able to carry out interpretation of the data and predict possible consequences.

For more detailed understanding of the idea mentioned above we present examples of all kinds of tasks from one of the mathematics courses: differential equations. It should be noted that in the future, engineers' training on this topic takes the leading position. This is due to the fact that, having arisen from problems of an applied nature, differential equations have been reflected in many sciences after further development of theories.

Therefore, the study of this topic contributes to the implementation of intersubject relationship. Secondly, it is in the process of learning the basics of the theory of differential equations that a student "... receives invaluable experience of real processes modeling" (Aslanov, 1997). During the study of differential equations, the review of a number of pedagogical and methodological studies helped to determine the following functions of mathematical modeling: cognitive, correction, interpretive. These functions have been implemented for solving tasks at all levels of difficulty.

Example 1. Algorithm: Solve an equation: $y^{\prime \prime}-y=e^{x}\left(x^{2}-1\right)$

In the process of solving the task students should know the basic concepts, facts and methods of the topic "Differential Equations"; to be able to solve differential equations and systems of differential equations of the simplest types; and, to know the methods of differential equations theory and mathematical modeling.

Example 2. Problem-research task: The body weight is $3 \mathrm{~kg}$ and the beginning it has a temperature. Ambient temperature is constant. Find the Newtonian cooling of the body.

It should be taken into account that this type of tasks can form an idea of the relationship of methods for solving differential equations with mathematical models of physical and chemical processes, as well as value-idea of the methods of solving 
differential equations for the future professional field, thereby contributing to students' interest in studying mathematics and sciences.

Example 3. Creative tasks: After switching off the engine the boat slows down its action caused by water resistance, which is proportional to the speed of the boat. The initial speed of the boat is $2 \mathrm{~m} / \mathrm{s}$, in Tc. its speed has been equal to $1 \mathrm{~m} / \mathrm{s}$. Draw the dependency graph of the boat's mileage from the start $(T)$ to a full stop. On the graph, mark possible intervals characterizing the properties of water (sea water, fresh water).

\section{RESULTS}

\section{Description of pilot testing}

Pilot testing was carried out on the basis of higher educational institution "Vyatka State University". The study involved 429 people. For the experiment, 2 groups were chosen: control (CG) and experimental (EG) groups. Pilot testing included three consecutive stages: preparatory, main and final.

In the preparatory phase there were implemented such tasks related to the development of information and methodological support, based on analysis of higher educational institution's work programs.

Analysis of psychological and educational literature and taking into account abovedescribed mathematical training functions (cognitive and applied) determined reasonability of realization of the following principles: fundamentality, practical orientation, informatization, greening and economization.

The principle of fundamentality is the key in the methodological culture formation of a professional and is to ensure consistency, coherence and scientific character of preparation process. The principle of practical orientation is aimed at resolving compliance problem of future careers' preparation with actual content of training. It is appropriate to draw attention to the ongoing scientific debate: fundamentality is a top priority, which is backed by theoretical basis and practical orientation (applied nature of engineering education). Following Livshits (2010), we believe that these priorities should not be opposing, but complementary.

The next principle of the organization and implementation of mathematical training of engineering students is the principle of informatization. It is caused by two main aspects. The first is defined by the terms of the modern information society, characterized by a continuous increase of information volume, a high level of information culture of competitive specialists, satisfaction of information needs through the use of modern information and communication technologies. The second aspect is directly connected with the intellectual component of engineering that involves the ability to work with the information and carry out its systematization, analysis, synthesis and generalization.

The principle of greening provides environmental focus of future engineers' training. This is due to the fact that the modern engineer must not only be able to undertake research, forecast and simulate the technical objects, but also to assess the environmental risks of his activities. The greening principle involves a consideration of the content of education from the standpoint of analysis, evaluation and forecasting of ecological safety of nature. Studies on the environmental component of future engineers' training emphasize that it is necessary to possess not only a safety culture, but also risk-oriented thinking. This approach is due to the fact that the conservation issues are considered as the most important priorities in professional work of the engineer.

The above principles are reflected in the organization of the preparatory stage of the study. At this stage, students become acquainted with the principles of operation with the system of tasks and with the system of learning outcomes' assessment. 
Setting and reasoning of learning objectives are carried out as well as development of students' cognitive interest in the mathematical disciplines, future profession. Besides, at this very stage there is a diagnostics of students' primary level of training, and there are set requirements for the level of professional knowledge assimilation and competence formation.

The teacher performs organizational, controlling, diagnostic functions; introduces students to the program of the course, the technology of information searching and processing, requirements to carry out written examinations in self-study, promotes the formation of individual educational direction, implemented at the main stage.

The main stage involves the assimilation of educational content, presented by a complex of theoretical and practical differentiated tasks. This step allows the teacher to manage the process of preparation, to coordinate independent work of students, together with students to make adjustments to follow individual educational direction, ensuring the continuity of the learning process. This allows you to organize the effective work of students with learning materials and implement formative assessment according to deadlines.

At the assessment and reflexive stage, there is generalization of knowledge and its application in practice, implementation of total control and reflection. For this purpose, the diagnostic component is used, comprising of differentiated tests, examinations and questions.

Each of the stages described involves an initial, current and final diagnostics. The aim of initial diagnostics is to study the features of students' readiness to training in university, the level of motivational component formation. This information is important for the subsequent organization of individual educational direction of every student. Current diagnostic tasks include identifying difficulties encountered by students in the study of mathematical disciplines, as well as tracking of the positive and negative dynamics of learning outcomes for the purpose of subsequent correction. The final diagnostics involves identifying the dynamics of the level of motivational component formation.

\section{The results of pilot testing}

This situation has led to a more detailed study of engineering students' motivational orientation. For this purpose, it was made a diagnostic study, aimed at determining the level of engineering students' motivation to study mathematics and science disciplines. To do this, we used diagnostics method of learning motivation orientation by Dubovitskaya (2002) and the method "The study of learning motivation in higher education".

Chart analysis enables us to conclude that there is a high percentage of students with low motivation to study mathematical disciplines.

Students of this group are generally not interested in the study of mathematics. They note that knowledge acquired in the classroom on these subjects is not likely to be useful for them in tackling life issues and professional problems. One of the key reasons for students' negative position is high level of abstraction of mathematical disciplines.

The average level of motivation was detected in $48.2 \%$ of the respondents. Students of this group emphasize the crucial role of mathematics for future career, and realize the importance of applied orientation of courses. They actively participated in the classes, commented and expressed their position in dealing with problem situations, and used additional literature for the fulfillment of assignments.

Students with a high level of motivation in studying mathematics and science disciplines (14.5\%) indicate a conscious choice of profession, interest to the study of subjects, the desire of enhanced training and constantly improve their knowledge and 
skills. Students of this group demonstrated a high level of activity, pace of work, and self-reliance.

The second method is "The study of learning motivation in higher education", which has allowed us to draw conclusions on the dominance of such motives as "graduation" and "getting the profession" among students $(44 \%$ and $37 \%$, respectively). Only $18 \%$ of respondents indicated that one of the main motives of university studies is to assimilate knowledge.

Thus, analyzing the data and student responses obtained in the course of diagnosis and real time conversations, it was found that reasons of insufficient high level of motivation to study mathematics are as follows: a high level of abstraction of educational material; the absence of its "focus" on the future professional activities; underdeveloped skills of organization and self-activity introspection, reflection, work with sources of information, its analysis and systematization.

After carrying out the experiment and its implementation in educational practice of tasks, we traced the dynamics of students' learning motivation of the control and experimental groups.

Diagram analysis highlights a positive trend in increasing the interest and awareness of mathematical disciplines' importance for students' future careers: number of students with a low level decreased from $36.3 \%$ to $18.6 \%$ due to the increase in the number of students having an average (from $48.0 \%$ to $59.3 \%$ ) and high $(15.7 \%$ to $22.1 \%)$ level of motivational component formation.

Thus, the objective of educational motivation development set at the ascertaining stage of the experiment was successfully carried out by the differentiated tasks implementation in educational practice.

\section{The factors leading to the development of learning motivation of students}

As a result of the work done we have highlighted the factors that ensure the development of learning motivation by solving differentiated tasks:

Motivational-and-adaptive: considering psycho-physiological characteristics and individual characteristics of the students; creation of stimulating situations ("situations of success", the introduction of elements of competition, etc.); ensuring the atmosphere of cooperation and dialogic interaction;

Subjective: the granting to students of the right to self-selected learning path with its subsequent correction; intensification of cognitive and learning activities through the provision of professional orientation training;

Integrative: integration of the content of math and sciences disciplines with elements of professional knowledge based on common concepts studied and interdisciplinary communication;

Diagnostic and regulatory: the implementation of the operational feedback; providing continuous pedagogical support of classroom and extracurricular work of students.

\section{DISCUSSION}

The problem of the development of learning motivation of students through the implementation of the system of tasks has been studied by many researchers. The view of Nikitina (2013) seem to be interesting as they developed a comprehensive program for active implementation of practice-oriented tasks. In particular, Mamayeva proposes the introduction of non-standard tasks and describes the mechanism of development of learning motivation of students, and proves the benefits of development of informative interest.

In accordance with the logic of our study, the position of Serikov (1999) is the most common. The author notes that introducing into the content of education emotionally- 
valuable, personal elements contributes to the development of inter-subject communication between all participants of the educational process, creates a favorable emotional environment and enhances the effectiveness of learning. Training material is perceived on a personal level, if it is experienced as a real-life task. This, in turn, "mobilizes and, accordingly, develops the structure of intelligence".

\section{CONCLUSION}

The proposed idea of the introduction into educational practices of the system of differentiated tasks is based on a personally-meaningful approach, which allows for the implementation of the principles of subjectivity, dialogism and individuality.

The result of the experimental work was the development of learning motivation of students through motivational-and-adaptive (consideration of psychophysiological peculiarities and individual characteristics of students, creation of stimulating situations (situations of success, the introduction of elements of competition, and others), ensuring an atmosphere of cooperation and dialogic interaction); subjective (granting to students the right to self-selected learning path with its subsequent correction; intensification of cognitive and learning activities through the provision of professional orientation training); integrative (integration of the content of math and sciences disciplines with elements of professional knowledge based on common concepts studied and interdisciplinary communication); diagnostic and regulatory (implementation of operational feedback; providing continuous pedagogical support of classroom and extracurricular work of students) possibilities of the system of differentiated tasks.

The promising lines of this problem research can be the use of information and communication technologies in teaching mathematical disciplines; establishment of research projects based on the implementation of interdisciplinary integration of mathematical and scientific disciplines.

\section{REFERENCES}

Aslanov, R.M. (1997) Methodical system of differential equations training in pedagogical school of higher education (Doctoral Thesis). Moscow.

Blonsky, P.P. (1979) Selected pedagogical and psychological works. Moscow: Pedagogika.

Bochkareva, O.V. (2006) Professional orientation in mathematics training for constructionengineering students in the school of higher education (Doctoral Thesis). Saransk.

Dubovitskaya, T.D. (2002) Psychological Science and Education. Diagnostic Method of Learning Motivation Direction, 2, 42-46.

Gladun, A.D. (2004) Laboratory workshop on general physics. Moscow: MIPT.

Hegel, G.F.V. (2005) Science of Logic. London: Science.

Khinchin, A.Y. (2000) on the educational phenomenon on lessons in mathematics. Mathematics in education and upbringing. Moscow: Phasis.

Leontiyv, A.A. (1996) Pedagogical dialogue. Moscow: "El- Fa."

Livshits V.I. (2010) Accreditation in Education. "Products" of Engineering Education: erudites or professionals? 6 (41), 13-19.

Markova, A.K. (2010) Formation of learning motivation at school age. Moscow: Prosvesheniye.

Mikhailova, I. G. (1998) The mathematical training of engineers in a professional orientation of interdisciplinary connections (Doctoral Thesis). Tobolsk.

Myshkis, A.D. (2007) Applied Mathematics for Engineers. Special courses. Moscow: FIZMATLIT.

Nikitina, A.L. (2013) TSU series "Humanities". Formation of professional competence by the construction and analysis of mathematical models of applied problems, 2, 447-457.

Serikov, V.V. (1999) Education and personality. The theory and practice of pedagogical systems' designing. Moscow: Logos.

Shchukina, G.I. (1979) Students' cognitive activity stimulation in the learning process. Moscow: Prosvesheniye. 
Teplov, B.M. (1985) Selected Works: Pedagogy. Moscow.

Tereshin, N.A. (1990). Applied orientation of school mathematics: Moscow: Prosvesheniye.

Utemov, V.V. (2012) Development of primary school pupils' creativity by solving open type tasks (Doctoral Thesis). Kirov.

Zimnyaya, I.A. (1999) Educational Psychology. Moscow: Logos.

$\diamond \diamond \diamond$ 